ESAIM: COCV

Vol. $13, \mathrm{~N}^{\mathrm{O}} 4$, 2007, pp. 692-706

DOI: $10.1051 /$ cocv:2007033
ESAIM: Control, Optimisation and Calculus of Variations

www.esaim-cocv.org

\title{
PARTIAL REGULARITY FOR ANISOTROPIC FUNCTIONALS OF HIGHER ORDER
}

\author{
Menita Carozza $^{1}$ And Antonia Passarelli di Napoli ${ }^{2}$
}

\begin{abstract}
We prove a $C^{k, \alpha}$ partial regularity result for local minimizers of variational integrals of the type $I(u)=\int_{\Omega} f\left(D^{k} u(x)\right) \mathrm{d} x$, assuming that the integrand $f$ satisfies $(p, q)$ growth conditions.
\end{abstract}

Mathematics Subject Classification. 35G99, 49N60, 49N99.

Received February 6, 2006. Revised June 8, 2006.

Published online July 20, 2007.

\section{INTRODUCTION}

Higher order variational functionals, emerging in the study of problems from materials science and engineering, have attracted a great deal of attention in last few years $[4,5,7]$. In particular, the regularity of minimizers of such functionals has been studied very recently. In [15] and [16] the partial $C^{k, \alpha}$ regularity has been established for quasiconvex integrals with a $p$-power growth with respect to the gradient and in [3] for convex integrals having subquadratic nonstandard growth condition, only in dimension 2 .

The aim of this paper is to establish the partial regularity of minimizers of integral functionals of the type

$$
I(u)=\int_{\Omega} f\left(D^{k} u(x)\right) \mathrm{d} x
$$

where $\Omega$ is a bounded subset of $\mathbb{R}^{n}, u: \Omega \subset \mathbb{R}^{n} \rightarrow \mathbb{R}^{N}, N \geq 1, k>1$ and $f$ is a $C^{2}$ convex integrand satisfying the non standard growth condition:

$$
C|\xi|^{p} \leq f(\xi) \leq L\left(1+|\xi|^{q}\right)
$$

with $p<q$, without restriction on the dimension and on the order of derivatives involved, in the superquadratic case.

Nonstandard growth conditions have been introduced by Marcellini, in the scalar case for $k=1$. He observed that, even in the scalar case, minimizers of (1) may fail to be regular (see $[13,17,18]$ ), when $q$ is too large with respect to $p$. On the other hand, one can prove regularity of scalar minimizers of (1) if $q$ is not too far away from $p$ (see e.g. [19] and its references). More precisely, in [19] it is shown that if one writes down the Euler equation for the functional $I$, under suitable assumptions on $p$ and $q$, the Moser iteration argument still works, thus leading to a sup estimate for the gradient $D u$ of the minimizer.

Keywords and phrases. Partial regularity, non standard growth, higher order derivatives.

1 Dipartimento Pe.Me.Is, Università degli studi del Sannio, Piazza Arechi 2, 82100 Benevento, Italy; carozza@unisannio.it

2 Dipartimento di Matematica e Applicazioni "R. Caccioppoli" Università di Napoli "Federico II", via Cintia, 80126 Napoli, Italy; antonia.passarelli@unina.it

(c) EDP Sciences, SMAI 2007 
Clearly this approach can not be carried on in the vector valued case, i.e. when $N>1$. First regularity results for systems are proved in [1] and [20] under special structure assumptions and in [22] in a more general setting. Moreover, higher integrability results for the gradient of the minimizers of (1) are avalaible in the vectorial case (see the references in $[2,8,9]$ ).

In this paper we prove that, for $k>1$, differently from all previous quoted results, if $f$ satisfies $(2)$ and the strong ellipticity assumption

$$
\left\langle D^{2} f(\xi) \eta, \eta\right\rangle \geq \gamma\left(1+|\xi|^{2}\right)^{\frac{p-2}{2}}|\eta|^{2}
$$

where

$$
2 \leq p<q<\min \left\{p+1, \frac{p n}{n-1}\right\}
$$

a minimizer $u \in W^{k, p}\left(\Omega ; \mathbb{R}^{N}\right)$ of functional (1) is $C^{k, \alpha}$ for all $\alpha<1$ in an open set $\Omega_{0} \subset \Omega$ such that $\operatorname{meas}\left(\Omega \backslash \Omega_{0}\right)=0$.

We point out that apart from condition (4), no special structure assumption is needed on $f$ and the condition on the exponents does not depend on $k$, i.e. the order of derivatives involved.

The proof of our result goes through a more or less standard blow-up argument aimed to establish a decay estimate on the excess function for the $k$ - order derivatives

$$
U\left(x_{0}, r\right)=f_{B_{r}\left(x_{0}\right)}\left|D^{k} u-\left(D^{k} u\right)_{x_{0}, r}\right|^{2}+\left|D^{k} u-\left(D^{k} u\right)_{x_{0}, r}\right|^{p} \mathrm{~d} x .
$$

Here, first order techniques have to be combined with new theoretical arguments needed to face the analytical and geometrical constraints of higher order derivatives. In particular, the essential tool is a lemma due to Fonseca and Malý (see [11] and also Lem. 2.4 below) which makes possible to connect in an annulus $B_{r} \backslash B_{s}$ two $W^{k, p}$ functions $v$ and $w$ with a more regular function function $z \in W^{k, q}\left(B_{r} \backslash B_{s}\right)$ with $p<q<\frac{p n}{n-1}$.

\section{Statements AND PRELiminary LEMmas}

Let us consider the functional

$$
I(u)=\int_{\Omega} f\left(D^{k} u(x)\right) \mathrm{d} x
$$

where $\Omega$ is a bounded open subset of $\mathbb{R}^{n}, n \geq 2$. Let $f: \mathbb{R}^{M N} \rightarrow \mathbb{R}$, where $M=\frac{(n+k-1) !}{k !(n-1) !}$ and $N \geq 2$, satisfy the following assumptions:

$$
\begin{gathered}
f \in C^{2} \\
C|\xi|^{p} \leq f(\xi) \leq L\left(1+|\xi|^{q}\right) \\
\left\langle D^{2} f(\xi) \eta, \eta\right\rangle \geq \gamma\left(1+|\xi|^{2}\right)^{\frac{p-2}{2}}|\eta|^{2}
\end{gathered}
$$

where

$$
2 \leq p<q<\min \left\{p+1, \frac{p n}{n-1}\right\}
$$

It is well known that

$$
|D f(\xi)| \leq c\left(1+|\xi|^{q-1}\right) .
$$

We say that $u \in W^{k, p}\left(\Omega, \mathbb{R}^{N}\right)$ is a minimizer of $I$ if

$$
I(u) \leq I(u+v)
$$

for any $v \in u+W_{0}^{k, p}\left(\Omega ; \mathbb{R}^{N}\right)$. 
Remark 2.1. If $u$ is a local minimizer of I and $\phi \in C_{0}^{k}\left(\Omega ; \mathbb{R}^{N}\right)$ from the minimality condition one has for any $\varepsilon>0$

$$
0 \leq \int_{\Omega}\left[f\left(D^{k} u+\varepsilon D^{k} \phi\right)-f\left(D^{k} u\right)\right] \mathrm{d} x=\varepsilon \int_{\Omega} \mathrm{d} x \int_{0}^{1} \frac{\partial f}{\partial \xi_{\alpha}^{i}}\left(D^{k} u+\varepsilon t D^{k} \phi\right) D_{\alpha} \phi^{i} \mathrm{~d} t
$$

where $|\alpha|=k$. Dividing this inequality by $\varepsilon$, and letting $\varepsilon$ go to zero, from (H4) and the assumption $q \leq p+1$ we get

$$
\int_{\Omega} \frac{\partial f}{\partial \xi_{\alpha}^{i}}\left(D^{k} u\right) D_{\alpha} \phi^{i} \mathrm{~d} x \geq 0
$$

and therefore, by the arbitrariness of $\phi$, the usual Euler-Lagrange system holds:

$$
\int_{\Omega} \frac{\partial f}{\partial \xi_{\alpha}^{i}}\left(D^{k} u\right) D_{\alpha} \phi^{i} \mathrm{~d} x=0 \quad \forall \phi \in C_{0}^{k}\left(\Omega ; \mathbb{R}^{N}\right)
$$

The aim of this paper is proving the following

Theorem 2.1. Let $f$ satisfy the assumptions $(\mathrm{H} 1)-(\mathrm{H} 3)$ and let $u \in W^{k, p}\left(\Omega ; \mathbb{R}^{N}\right)$ be a minimizer of $I$. Then there exists an open subset $\Omega_{0}$ of $\Omega$ such that

$$
\operatorname{meas}\left(\Omega \backslash \Omega_{0}\right)=0
$$

and

$$
u \in C^{k, \alpha}\left(\Omega_{0}, \mathbb{R}^{N}\right) \quad \text { for all } \quad \alpha<1 .
$$

In what follows, we will denote by $u$ a $W^{k, p}\left(\Omega ; \mathbb{R}^{N}\right)$ minimizer of the integral functional (1) and assume that its integrand $f$ satisfies $(\mathrm{H} 1)-(\mathrm{H} 3)$. We set for every $B_{r}\left(x_{0}\right) \subset \Omega$

$$
f_{B_{r}\left(x_{0}\right)} g=(g)_{x_{0}, r}=\frac{1}{\operatorname{meas}\left(B_{r}\left(x_{0}\right)\right)} \int_{B_{r}\left(x_{0}\right)} g .
$$

Moreover, given $p>1$ and $u \in W^{k, p}\left(\Omega ; \mathbb{R}^{N}\right), k \geq 1$, we will denote by $P(y)=P_{u}(x, R, y)$ the unique polynomial of degree $k-1$ such that

$$
\int_{B_{r}(x)} D^{l}(u(y)-P(y)) \mathrm{d} y=0 \quad l=1, \ldots, k-1 .
$$

Its coefficients depend on $x, R$ and also on the derivatives of $u$ (see [12]). When no confusion will arise, we will omit the dependence of $P$ on $x, R$ and $u$.

Next lemma can be found in [11], (Th. 3.3), in a slightly different form.

Lemma 2.2. Let $v \in W^{k, p}\left(B_{1}(0)\right)$ and $0<s<r<1$. There exists a linear operator $T: W^{k, p}\left(B_{1}(0)\right) \rightarrow$ $W^{k, p}\left(B_{1}(0)\right)$ such that

$$
T v=v \quad \text { on } \quad\left(B_{1} \backslash B_{r}\right) \cup B_{s}
$$

and for all $\mu>0$, for all $q<p \frac{n}{n-1}$ 


$$
\begin{aligned}
\left(\int_{B_{r} \backslash B_{s}}\left|D^{k} T v\right|^{2}\right)^{\frac{1}{2}}+ & \mu\left(\int_{B_{r} \backslash B_{s}}\left|D^{k} T v\right|^{q}\right)^{\frac{1}{q}} \\
\leq C(r-s)^{\rho}\{ & {\left[\sup _{t \in(s, r)}(t-s)^{-\frac{1}{2}}\left(\int_{B_{t} \backslash B_{s}}\left|D^{k} v\right|^{2}\right)^{\frac{1}{2}}+\sup _{t \in(s, r)}(r-t)^{-\frac{1}{2}}\left(\int_{B_{t} \backslash B_{s}}\left|D^{k} v\right|^{2}\right)^{\frac{1}{2}}\right] } \\
+ & \left.\mu\left[\sup _{t \in(s, r)}(t-s)^{-\frac{1}{p}}\left(\int_{B_{r} \backslash B_{t}}\left|D^{k} v\right|^{p}\right)^{\frac{1}{p}}+\sup _{t \in(s, r)}(r-t)^{-\frac{1}{p}}\left(\int_{B_{r} \backslash B_{t}}\left|D^{k} v\right|^{p}\right)^{\frac{1}{p}}\right]\right\}
\end{aligned}
$$

where $C=C(n, p, q)>0$, and $\rho=\rho(n, p, q)>0$.

Let us recall an elementary Lemma proved in [10].

Lemma 2.3. Let $\psi$ be a continuous nondecreasing function on an interval $[a, b], a<b$. There exist $a^{\prime} \in$ $\left[a, a+\frac{1}{3}(b-a)\right], b^{\prime} \in\left[b-\frac{1}{3}(b-a), b\right]$ such that $a \leq a^{\prime}<b^{\prime} \leq b$ and

$$
\begin{aligned}
& \frac{\psi(t)-\psi\left(a^{\prime}\right)}{t-a^{\prime}} \leq 3 \frac{(\psi(b)-\psi(a))}{b-a} \\
& \frac{\psi\left(b^{\prime}\right)-\psi(t)}{b^{\prime}-t} \leq 3 \frac{(\psi(b)-\psi(a))}{b-a}
\end{aligned}
$$

for all $t \in\left(a^{\prime}, b^{\prime}\right)$.

Finally, combining the previous two lemmas we obtain a generalization to the case of higher order derivatives of Lemma 2.4 in [10]. We give the proof here for completeness.

Lemma 2.4. Let $v, w \in W^{k, p}\left(B_{1}(0)\right)$ and $\frac{1}{4}<s<r<1$. Fix $p<q<\frac{n p}{n-1}$, for all $\mu>0$ and $m \in$ IN there exist a function $z \in W^{k, p}\left(B_{1}(0)\right)$ and $\frac{1}{4}<s<s^{\prime}<r^{\prime}<r<1$ with $r^{\prime}$, $s^{\prime}$ depending on $v$, $w$ and $\mu$, such that

$$
\begin{gathered}
z=v \quad \text { on } \quad B_{s^{\prime}}, \quad z=w \quad \text { on } \quad B_{1} \backslash B_{r^{\prime}}, \\
\frac{r-s}{m} \geq r^{\prime}-s^{\prime} \geq \frac{r-s}{3 m}
\end{gathered}
$$

and

$$
\begin{aligned}
& \left(\int_{B_{r^{\prime}} \backslash B_{s^{\prime}}}\left|D^{k} z\right|^{2}\right)^{\frac{1}{2}}+\mu\left(\int_{B_{r^{\prime}} \backslash B_{s^{\prime}}}\left|D^{k} z\right|^{q}\right)^{\frac{1}{q}} \\
& \leq C \frac{(r-s)^{\rho}}{m^{\rho}}\left[f_{B_{r} \backslash B_{s}}\left(1+\sum_{l=0}^{k}\left|D^{l} v\right|^{2}+\sum_{l=0}^{k}\left|D^{l} w\right|^{2}+\frac{m^{2}}{(r-s)^{2}} \sum_{l=1}^{k-1}\left|D^{l}(v-w)\right|^{2}\right)\right. \\
& \left.+\mu^{p} f_{B_{r} \backslash B_{s}}\left(1+\sum_{l=0}^{k}\left|D^{l} v\right|^{p}+\sum_{l=1}^{k}\left|D^{l} w\right|^{p}+\frac{m^{p}}{(r-s)^{p}} \sum_{l=0}^{k-1}\left|D^{l}(v-w)\right|^{p}\right)\right]^{\frac{1}{2}}
\end{aligned}
$$

where $C=C(n, p, q)>0$ and $\rho=\rho(p, q, n)>0$. 
Proof. As in Lemma 2.4 in [10], choose $m \in I N$ and set

$$
\begin{gathered}
f=1+\sum_{l=0}^{k}\left|D^{l} v\right|^{2}+\sum_{l=0}^{k}\left|D^{l} w\right|^{2}+\frac{m^{2}}{(r-s)^{2}} \sum_{l=0}^{k-1}\left|D^{l}(v-w)\right|^{2} \\
+\mu^{p}\left(1+\sum_{l=0}^{k}\left|D^{l} v\right|^{p}+\sum_{l=0}^{k}\left|D^{l} w\right|^{p}+\frac{m^{p}}{(r-s)^{p}} \sum_{l=0}^{k-1}\left|D^{l}(v-w)\right|^{p}\right) .
\end{gathered}
$$

We may find $h \in\{1, \ldots, m\}$ such that

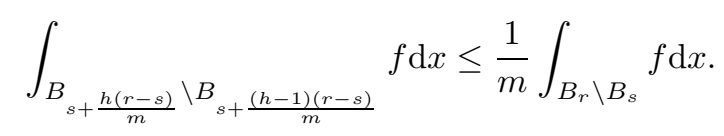

Set, for $t \in\left[s+\frac{(h-1)(r-s)}{m}, s+\frac{h(r-s)}{m}\right]$,

$$
\psi(t)=\int_{B_{t} \backslash B_{s}} f \mathrm{~d} x
$$

which is a continuous increasing function. By Lemma 2.3, there exists $\left[s^{\prime}, r^{\prime}\right] \subset\left[s+\frac{(h-1)(r-s)}{m}, s+\frac{h(r-s)}{m}\right]$ such that

and

$$
\frac{r-s}{m} \geq r^{\prime}-s^{\prime} \geq \frac{r-s}{3 m}
$$

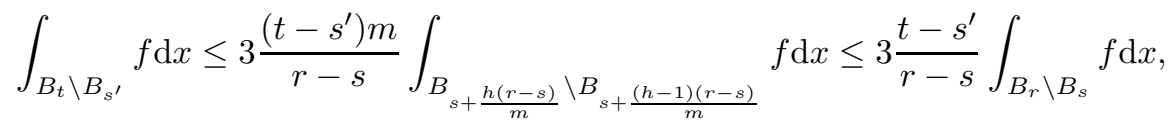

$$
\begin{aligned}
& \int_{B_{r^{\prime}} \backslash B_{t}} f \mathrm{~d} x \leq 3 \frac{r^{\prime}-t}{r-s} \int_{B_{r} \backslash B_{s}} f \mathrm{~d} x
\end{aligned}
$$

for all $t \in\left(s^{\prime}, r^{\prime}\right)$. Set

A direct computation shows that

$$
u=\left\{\begin{array}{lll}
v(x) & \text { if } \quad x \in B_{s^{\prime}} \\
\frac{\left(r^{\prime}-|x|\right) v(x)+\left(|x|-s^{\prime}\right) w(x)}{r^{\prime}-s^{\prime}} & \text { if } \quad x \in B_{r^{\prime}} \backslash B_{s^{\prime}} \\
w(x) & \text { if } \quad x \in B_{1} \backslash B_{r^{\prime}} .
\end{array}\right.
$$

$$
\sum_{l=0}^{k}\left|D^{l} u\right|^{2}+\mu^{q}\left(\sum_{l=0}^{k}\left|D^{l} u\right|^{p}\right) \leq C f .
$$

If we apply Lemma 2.2 to the function $u$, we then find $z \in W^{k, p}\left(B_{1}\right)$ satisfying (6). Moreover, from (8) and (9), using (5), one readily cheks that

$$
\begin{aligned}
&\left(\int_{B_{r^{\prime}} \backslash B_{s^{\prime}}}\left|D^{k} z\right|^{2}\right)^{\frac{1}{2}}+\mu\left(\int_{B_{r^{\prime}} \backslash B_{s^{\prime}}}\left|D^{k} z\right|^{q}\right)^{\frac{1}{q}} \\
& \leq c\left(r^{\prime}-s^{\prime}\right)^{\rho}\left\{\frac{\left|B_{r^{\prime}} \backslash B_{s^{\prime}}\right|^{\frac{1}{2}}}{\left(r^{\prime}-s^{\prime}\right)^{\frac{1}{2}}}\left(f_{B_{r} \backslash B_{s}} f\right)^{\frac{1}{2}}+\frac{\left|B_{r^{\prime}} \backslash B_{s^{\prime}}\right|^{\frac{1}{p}}}{\left(r^{\prime}-s^{\prime}\right)^{\frac{1}{p}}}\left(f_{B_{r} \backslash B_{s}} f\right)^{\frac{1}{p}}\right\} \\
& \quad \leq c\left(r^{\prime}-s^{\prime}\right)^{\rho}\left\{\left(f_{B_{r} \backslash B_{s}} f\right)^{\frac{1}{2}}+\left(f_{B_{r} \backslash B_{s}} f\right)^{\frac{1}{p}}\right\},
\end{aligned}
$$

from which (7) follows. 


\section{The DeCay estimate}

As usual, to get the partial regularity result stated in Theorem 2.1, we need a decay estimate for the excess function $U\left(x_{0}, r\right)$ defined as follows

$$
U\left(x_{0}, r\right)=f_{B_{r\left(x_{0}\right)}}\left[\left|D^{k} u-\left(D^{k} u\right)_{x_{0}, r}\right|^{2}+\left|D^{k} u-\left(D^{k} u\right)_{x_{0}, r}\right|^{p}\right] \mathrm{d} y
$$

which measures how the $k$-order derivatives are far from being constant in the ball $B_{r\left(x_{0}\right)}$. The desired decay estimate is established in the next proposition.

Proposition 3.1. Fix $M>0$. There exists a constant $C_{M}>0$ such that for every $0<\tau<\frac{1}{4}$, there exists $\epsilon=\epsilon(\tau, M)$ such that, if

then

$$
\left|\left(D^{k} u\right)_{x_{0}, r}\right| \leq M \quad \text { and } \quad U\left(x_{0}, r\right) \leq \epsilon
$$

$$
U\left(x_{0}, \tau r\right) \leq C_{M} \tau^{2} U\left(x_{0}, r\right)
$$

Proof. Fix M and $\tau$. We shall determine $C_{M}$ later. We argue by contradiction assuming that there exists a sequence $B_{r_{h}}\left(x_{h}\right)$ satisfying

$$
B_{r_{h}}\left(x_{h}\right) \subset \Omega, \quad\left|\left(D^{k} u\right)_{x_{h}, r_{h}}\right| \leq M, \quad \lim _{h} U\left(x_{h}, r_{h}\right)=0,
$$

but

$$
U\left(x_{h}, \tau r_{h}\right)>C_{M} \tau^{2} U\left(x_{h}, r_{h}\right)
$$

Set

$$
A_{h}=\left(D^{k} u\right)_{x_{h}, r_{h}} \quad \lambda_{h}^{2}=U\left(x_{h}, r_{h}\right)
$$

and let $P$ the polynomial such that

$$
\int_{B_{r_{h}}\left(x_{h}\right)} D^{l}(u-P)=0 \quad l=0, \ldots, k
$$

Step 1. Blow up. We rescale the function $u$ in each $B_{r_{h}}\left(x_{h}\right)$ to obtain a sequence of functions on $B_{1}(0)$. Set

$$
v_{h}(y)=\frac{1}{\lambda_{h} r_{h}^{k}}\left[u\left(x_{h}+r_{h} y\right)-P\left(x_{h}+r_{h} y\right)\right],
$$

then

Clearly we have

$$
D^{k} v_{h}(y)=\frac{1}{\lambda_{h}}\left[D^{k} u\left(x_{h}+r_{h} y\right)-A_{h}\right]
$$

Moreover,

$$
\left(D^{l} v_{h}\right)_{0,1}=0 \quad l=0, \ldots, k .
$$

$$
\frac{U\left(x_{h}, r_{h}\right)}{\lambda_{h}^{2}}=f_{B_{1}}\left[\left|D^{k} v_{h}\right|^{2}+\lambda_{h}^{p-2}\left|D^{k} v_{h}\right|^{p}\right] \mathrm{d} y=1 .
$$

Then, passing possibly to a subsequence, we may suppose that

$$
v_{h} \rightarrow v \quad \text { weakly in } W^{k, 2}\left(B_{1} ; \mathbb{R}^{N}\right)
$$


and, since $\forall h \quad\left|A_{h}\right| \leq M$,

$$
A_{h} \rightarrow A \text {. }
$$

Step 2. $v$ solves $a$ linear system. Now we show that

$$
\int_{B_{1}(0)} \frac{\partial^{2} f}{\partial \xi_{\alpha}^{i} \partial \xi_{\beta}^{j}}(A) D_{\beta} v^{j} D_{\alpha} \phi^{i} \mathrm{~d} y=0 \quad \forall \phi \in C_{0}^{k}\left(B_{1} ; \mathbb{R}^{N}\right)
$$

Since we assume $q-1 \leq p$ we can write the usual Euler-Lagrange system for $u$ (see Rem. 2.1). Then, rescaling in each $B_{r_{h}}\left(x_{h}\right)$, we get for any $\phi \in C_{0}^{k}\left(B_{1} ; \mathbb{R}^{N}\right)$ and any $1 \leq i \leq N$

$$
\int_{B_{1}(0)} \frac{\partial f}{\partial \xi_{\alpha}^{i}}\left(A_{h}+\lambda_{h} D^{k} v_{h}\right) D_{\alpha} \phi^{i} \mathrm{~d} y=0
$$

where $|\alpha|=k$. Then

$$
\frac{1}{\lambda_{h}} \int_{B_{1}(0)}\left[\frac{\partial f}{\partial \xi_{\alpha}^{i}}\left(A_{h}+\lambda_{h} D^{k} v_{h}\right)-\frac{\partial f}{\partial \xi_{\alpha}^{i}}\left(A_{h}\right)\right] D_{\alpha} \phi^{i} \mathrm{~d} y=0 .
$$

Let us split

$$
B_{1}=E_{h}^{+} \cup E_{h}^{-}=\left\{y \in B_{1}: \lambda_{h}\left|D^{k} v_{h}(y)\right|>1\right\} \cup\left\{y \in B_{1}: \lambda_{h}\left|D^{k} v_{h}(y)\right| \leq 1\right\}
$$

then, by (11), we get

$$
\left|E_{h}^{+}\right| \leq \int_{E_{h}^{+}} \lambda_{h}^{2}\left|D^{k} v_{h}\right|^{2} \mathrm{~d} y \leq \lambda_{h}^{2} \int_{B_{1}(0)}\left|D^{k} v_{h}\right|^{2} \mathrm{~d} y \leq c \lambda_{h}^{2} .
$$

Now, by (H4) and Hölder's inequality, we observe that

$$
\begin{aligned}
\frac{1}{\lambda_{h}}\left|\int_{E_{h}^{+}}\left[D f\left(A_{h}+\lambda_{h} D^{k} v_{h}\right)-D f\left(A_{h}\right)\right] D \phi \mathrm{d} y\right| & \\
\leq \frac{c}{\lambda_{h}}\left|E_{h}^{+}\right|+c \lambda_{h}^{q-2} \int_{E_{h}^{+}}\left|D^{k} v_{h}\right|^{q-1} \mathrm{~d} y & \leq c \lambda_{h}+c\left(\int_{E_{h}^{+}} \lambda_{h}^{p-2}\left|D^{k} v_{h}\right|^{p} \mathrm{~d} y\right)^{\frac{q-1}{p}} \lambda_{h}^{\frac{2 q-p-2}{p}}\left|E_{h}^{+}\right|^{\frac{p-q+1}{p}} \leq c \lambda_{h}
\end{aligned}
$$

where we used again the assumption $q-1 \leq p$.

From this it follows that

$$
\lim _{h} \frac{1}{\lambda_{h}} \int_{E_{h}^{+}}\left[D f\left(A_{h}+\lambda_{h} D^{k} v_{h}\right)-D f\left(A_{h}\right)\right] D \phi \mathrm{d} y=0 .
$$

On $E_{h}^{-}$we have

$$
\begin{aligned}
\frac{1}{\lambda_{h}} \int_{E_{h}^{-}}\left[D f\left(A_{h}+\lambda_{h} D^{k} v_{h}\right)-D f\left(A_{h}\right)\right] D \phi \mathrm{d} y & =\int_{E_{h}^{-}} \int_{0}^{1} D^{2} f\left(A_{h}+s \lambda_{h} D^{k} v_{h}\right) D^{k} v_{h} D \phi \mathrm{d} s \mathrm{~d} y \\
& =\int_{E_{h}^{-}} \int_{0}^{1}\left[D^{2} f\left(A_{h}+s \lambda_{h} D^{k} v_{h}\right)-D^{2} f\left(A_{h}\right)\right] D^{k} v_{h} D \phi \mathrm{d} s \mathrm{~d} y \\
& +\int_{E_{h}^{-}} D^{2} f\left(A_{h}\right) D^{k} v_{h} D \phi \mathrm{d} y .
\end{aligned}
$$


Note that (16) ensures that $\chi_{E_{h}^{-}} \rightarrow \chi_{B_{1}}$ in $L^{r}\left(B_{1}\right)$ for all $r<\infty$ and by (11) we have, passing possibly to a subsequence,

$$
\lambda_{h} D^{k} v_{h}(y) \rightarrow 0 \quad \text { a.e. } \quad \text { in } \quad B_{1}
$$

Then, by (12), (13) and the uniform continuity of $D^{2} f$ on bounded sets, we get

$$
\lim _{h} \frac{1}{\lambda_{h}} \int_{E_{h}^{-}}\left[D f\left(A_{h}+\lambda_{h} D^{k} v_{h}\right)-D f\left(A_{h}\right)\right] D \phi \mathrm{d} y=\int_{B_{1}} D^{2} f(A) D^{k} v D \phi \mathrm{d} y .
$$

Collecting (15), (17) and the above equality, we obtain that $v$ satisfies system (14), which is linear and elliptic with constant coefficients by (H3). By standard regularity results (see [12]), we have for any $0<\tau<1$

$$
f_{B_{\tau}}\left|D^{k} v-\left(D^{k} v\right)_{\tau}\right|^{2} \mathrm{~d} y \leq c \tau^{2} f_{B_{1}}\left|D^{k} v-\left(D^{k} v\right)_{1}\right|^{2} \mathrm{~d} y \leq c \tau^{2}
$$

Moreover we have

$$
v \in C^{\infty}\left(B_{1} ; \mathbb{R}^{N}\right)
$$

and

$$
\lambda_{h}^{\frac{p-2}{p}}\left(v_{h}-v\right) \rightarrow 0 \quad \text { weakly in } W_{\text {loc }}^{k, p}\left(B_{1} ; \mathbb{R}^{N}\right)
$$

Step 3. Upper bound. We set

$$
f_{h}(\xi)=\frac{1}{\lambda_{h}^{2}}\left[f\left(A_{h}+\lambda_{h} \xi\right)-f\left(A_{h}\right)-\lambda_{h} \operatorname{Df}\left(A_{h}\right) \xi\right]
$$

and, for every $r<1$, we consider

$$
I_{h, r}(w)=\int_{B_{r}} f_{h}\left(D^{k} w\right) \mathrm{d} y
$$

Note that, by the strong ellipticity assumption (H3), it follows that $f_{h}(\xi) \geq 0$, for any $\xi$, and remember that $v_{h}$ is a local minimizer for each $I_{h, r}$. Fix $\frac{1}{4}<s<1$. Passing to a subsequence we may always assume that

$$
\lim _{h}\left[I_{h, s}\left(v_{h}\right)-I_{h, s}(v)\right]
$$

exists.

We shall prove that

$$
\lim _{h}\left[I_{h, s}\left(v_{h}\right)-I_{h, s}(v)\right] \leq 0
$$

Consider $r>s$ and fix $m \in I N$. Observe that, since $v \in W^{k, p}\left(B_{1}\right)$ and $v_{h} \in W^{k, p}\left(B_{1}\right)$, Lemma 2.4, with $\mu=\lambda_{h}^{\frac{q-2}{q}}$, implies that there exist $z_{h} \in W^{k, p}\left(B_{1}\right)$ and $\frac{1}{4}<s<s_{h}<r_{h}<r<1$ such that

$$
z_{h}=v \quad \text { on } \quad B_{s_{h}} \quad z_{h}=v_{h} \quad \text { on } \quad B_{1} \backslash B_{r_{h}}
$$


and

$$
\begin{aligned}
\left(\int_{B_{r_{h}} \backslash B_{s_{h}}}\left|D^{k} z_{h}\right|^{2}\right)^{\frac{1}{2}}+\lambda_{h}^{\frac{q-2}{q}} & \left(\int_{\left.B_{r_{h} \backslash B_{s_{h}}}\left|D^{k} z_{h}\right|^{q}\right)^{\frac{1}{q}}}\right. \\
\leq C \frac{(r-s)^{\rho}}{m^{\rho}} & {\left[f_{B_{r} \backslash B_{s}}\left(1+\sum_{l=0}^{k}\left|D^{l} v\right|^{2}+\sum_{l=0}^{k}\left|D^{l} v_{h}\right|^{2}+\frac{m^{2}}{(r-s)^{2}} \sum_{l=1}^{k-1}\left|D^{l}\left(v-v_{h}\right)\right|^{2}\right)\right.} \\
& \left.+\lambda_{h}^{\frac{q-2}{q} p} f_{B_{r} \backslash B_{s}}\left(1+\sum_{l=0}^{k}\left|D^{l} v\right|^{p}+\sum_{l=0}^{k}\left|D^{l} v_{h}\right|^{p}+\frac{m^{p}}{(r-s)^{p}} \sum_{l=1}^{k-1}\left|D^{l}\left(v-v_{h}\right)\right|^{p}\right)\right]^{\frac{1}{2}}
\end{aligned}
$$

Since by (19), $D^{k} v$ is locally bounded on $B_{1}$ we get

$$
\begin{aligned}
I_{h, s}\left(v_{h}\right)-I_{h, s}(v) & \leq I_{h, r_{h}}\left(v_{h}\right)-I_{h, r_{h}}(v)+I_{h, r_{h}}(v)-I_{h, s}(v) \\
& =I_{h, r_{h}}\left(v_{h}\right)-I_{h, r_{h}}(v)+\int_{B_{r_{h} \backslash B_{s}}} f_{h}\left(D^{k} v\right) \\
& \leq I_{h, r_{h}}\left(z_{h}\right)-I_{h, r_{h}}(v)+c(r-s) \\
& \leq \int_{B_{r_{h}} \backslash B_{s_{h}}}\left[f_{h}\left(D^{k} z_{h}\right)-f_{h}\left(D^{k} v\right)\right]+c(r-s)
\end{aligned}
$$

where we used the minimality of $v_{h}$.

As $\left|f_{h}(\xi)\right| \leq c\left(|\xi|^{2}+\lambda_{h}^{q-2}|\xi|^{q}\right)$, we get by (21), using the fact that $\frac{r-s}{m}<1$ and that the quantity on square brackets is greater or equal than 1 ,

$$
\begin{aligned}
I_{h, r_{h}}\left(z_{h}\right)-I_{h, r_{h}}(v) & \leq c \int_{B_{r_{h}} \backslash B_{s_{h}}}\left|D^{k} z_{h}\right|^{2}+\lambda_{h}^{q-2}\left|D^{k} z_{h}\right|^{q} \\
& \leq c \frac{(r-s)^{2 \rho}}{m^{2 \rho}}\left[f_{B_{r} \backslash B_{s}}\left(1+\sum_{l=0}^{k}\left|D^{l} v\right|^{2}+\sum_{l=0}^{k}\left|D^{l} v_{h}\right|^{2}+\frac{m^{2}}{(r-s)^{2}} \sum_{l=1}^{k-1}\left|D^{l}\left(v-v_{h}\right)\right|^{2}\right)\right]^{\frac{q}{2}} \\
& +c \frac{(r-s)^{2 \rho}}{m^{2 \rho}}\left[\lambda_{h}^{\frac{q-2}{q} p} f_{B_{r} \backslash B_{s}}\left(1+\sum_{l=0}^{k}\left|D^{l} v\right|^{p}+\sum_{l=0}^{k}\left|D^{l} v_{h}\right|^{p}+\frac{m^{p}}{(r-s)^{p}} \sum_{l=1}^{k-1}\left|D^{l}\left(v-v_{h}\right)\right|^{p}\right)\right]^{\frac{q}{2}} \\
& =J_{h, 1}+J_{h, 2} .
\end{aligned}
$$

Since $D^{l} v_{h} \rightarrow D^{l} v$ strongly in $L^{2}\left(B_{1} ; \mathbb{R}^{N}\right)$ for every $l<k$, we have, using (11)

$$
\limsup _{h \rightarrow \infty} J_{h, 1} \leq c m^{-2 \rho} .
$$

Moreover, since for $l=0, \ldots, k$

$$
\lambda_{h}^{\frac{p(q-2)}{q}} \int_{B_{1}}\left|D^{l} v_{h}\right|^{p} \leq c \lambda_{h}^{\frac{2(q-p)}{q}} \lambda_{h}^{p-2} \int_{B_{1}}\left|D^{k} v_{h}\right|^{p} \leq c \lambda_{h}^{\frac{2(q-p)}{q}}
$$

and

we have

$$
\lambda_{h}^{\frac{p(q-2)}{q}} \int_{B_{1}}\left|D^{l}\left(v_{h}-v\right)\right|^{p} \leq c \lambda_{h}^{\frac{p(q-2)}{q}} \int_{B_{1}}\left|D^{k} v_{h}\right|^{p} \leq c \lambda_{h}^{\frac{2(q-p)}{q}}
$$

$\lim _{h} J_{h, 2}=0$ 
Hence we conclude letting first $m \rightarrow \infty$ and then $r \rightarrow s$ in (22).

Step 4. Lower bound. We shall prove that, for a.e. $\frac{1}{4}<r<\frac{1}{2}$, if $t<r$ then

$$
\underset{h}{\limsup } \int_{B_{t}}\left|D^{k} v-D^{k} v_{h}\right|^{2}\left(1+\lambda_{h}^{p-2}\left|D^{k} v-D^{k} v_{h}\right|^{p-2}\right) \leq \lim _{h}\left[I_{h, r}\left(v_{h}\right)-I_{h, r}(v)\right] .
$$

For any Borel set $A \subset B_{1}$, let us define

$$
\mu_{h}(A)=\int_{A} \sum_{l=0}^{k}\left|D^{l} v_{h}\right|^{2} \mathrm{~d} x
$$

Passing possibly to a subsequence, since $\mu_{h}\left(B_{1}\right) \leq c$, we may suppose

$$
\mu_{h} \rightarrow \mu \quad \text { weakly } * \text { in the sense of measures, }
$$

where $\mu$ is a Borel measure over $B_{1}$, with finite total variation. Then for a.e. $r<1$

$$
\mu\left(\partial B_{r}\right)=0
$$

and let us choose such a radius $r$. Consider $\frac{1}{4}<t<s<r$, also such that $\mu\left(\partial B_{s}\right)=0$, and fix $m \in I N$. Observe that, as $v_{h} \in W^{k, p}\left(B_{1}\right)$ Lemma 2.4 implies that there exist $z_{h} \in W^{k, p}\left(B_{1}\right)$ and $\frac{1}{4}<s<s_{h}<r_{h}<r<1$ such that

and

$$
\begin{gathered}
z_{h}=v_{h} \quad \text { on } \quad B_{s_{h}} \quad z_{h}=v_{h} \quad \text { on } \quad B_{1} \backslash B_{r_{h}} \\
r_{h}-s_{h} \geq \frac{r-s}{3 m}
\end{gathered}
$$

$$
\begin{aligned}
&\left(\int_{B_{r_{h}} \backslash B_{s_{h}}}\left|D^{k} z_{h}\right|^{2}\right)^{\frac{1}{2}}+\lambda_{h}^{\frac{q-2}{q}}\left(\int_{B_{r_{h}} \backslash B_{s_{h}}}\left|D^{k} z_{h}\right|^{q}\right)^{\frac{1}{q}} \\
& \leq C \frac{(r-s)^{\rho}}{m^{\rho}}\left[f_{B_{r} \backslash B_{s}}\left(1+\sum_{l=0}^{k}\left|D^{l} v_{h}\right|^{2}\right)+\lambda_{h}^{\frac{(q-2) p}{q}} f_{B_{r} \backslash B_{s}}\left(1+\sum_{l=0}^{k}\left|D^{l} v_{h}\right|^{p}\right)\right]^{\frac{1}{2}}
\end{aligned}
$$

Passing possibly to a subsequence, we may suppose that

$$
z_{h} \rightarrow v_{r, s} \quad \text { weakly in } W^{k, 2}\left(B_{1}\right)
$$

and

$$
v_{r, s}=v \quad \text { in } \quad\left(B_{1} \backslash B_{r}\right) \cup B_{s} .
$$

Moreover, from (23) and the interpolation inequality with $\frac{1}{p}=\frac{\theta}{2}+\frac{1-\theta}{q}$, we deduce that

$$
\begin{aligned}
\lambda_{h}^{p-2} \int_{B_{1}}\left|D^{k} z_{h}\right|^{p} & \leq c \lambda_{h}^{p-2}\left(\int_{B_{1}}\left|D^{k} z_{h}\right|^{2}\right)^{\frac{\theta p}{2}}\left(\int_{B_{1}}\left|D^{k} z_{h}\right|^{q}\right)^{\frac{(1-\theta) p}{2}} \\
& \leq c \lambda_{h}^{p-2}\left(\int_{B_{1}}\left|D^{k} z_{h}\right|^{q}\right)^{\frac{(1-\theta) p}{2}} \\
& \leq c \lambda_{h}^{p-2}\left(c \lambda_{h}^{2-q}\right)^{\frac{p-2}{q-2}} \leq c
\end{aligned}
$$


since $\theta=\frac{p-2}{q-2} \frac{q}{p}$.

Consider $\zeta_{h} \in C_{0}^{\infty}\left(B_{r_{h}}\right)$ such that $0 \leq \zeta_{h} \leq 1, \zeta_{h}=1$ on $B_{s_{h}}$ and $\left|D^{l} \zeta_{h}\right| \leq \frac{C}{\left(r_{h}-s_{h}\right)^{2}}$, for $l=0, \ldots, k$, and set

$$
\psi_{h}^{\epsilon}=\zeta_{h}\left(z_{h}-v_{r, s}^{\epsilon}\right)
$$

where $v_{r, s}^{\epsilon}=\rho_{\epsilon} \star v_{r, s}$, and $\rho_{\epsilon}$ is the usual sequence of mollifiers. Now, setting $v^{\epsilon}=\rho_{\epsilon} \star v$, we observe that

$$
\begin{aligned}
I_{h, r_{h}}\left(v_{h}\right)-I_{h, r_{h}}\left(v^{\epsilon}\right)= & I_{h, r_{h}}\left(v_{h}\right)-I_{h, r_{h}}\left(z_{h}\right)+I_{h, r_{h}}\left(z_{h}\right)-I_{h, r_{h}}\left(v_{r, s}^{\epsilon}+\psi_{h}^{\epsilon}\right) \\
& +I_{h, r_{h}}\left(\psi_{h}^{\epsilon}+v_{r, s}^{\epsilon}\right)-I_{h, r_{h}}\left(v_{r, s}^{\epsilon}\right)-I_{h, r_{h}}\left(\psi_{h}^{\epsilon}\right)+I_{h, r_{h}}\left(v_{r, s}^{\epsilon}\right)-I_{h, r_{h}}\left(v^{\epsilon}\right) \\
& +I_{h, r_{h}}\left(\psi_{h}^{\epsilon}\right) \\
= & R_{h, 1}+R_{h, 2}+R_{h, 3}+R_{h, 4}+R_{h, 5} .
\end{aligned}
$$

To bound $R_{h, 1}$ we observe that

$$
I_{h, r_{h}}\left(v_{h}\right)-I_{h, r_{h}}\left(z_{h}\right)=\int_{B_{r_{h}} \backslash B_{s_{h}}} f_{h}\left(D^{k} v_{h}\right)-\int_{B_{r_{h}} \backslash B_{s_{h}}} f_{h}\left(D^{k} z_{h}\right) \geq-\int_{B_{r_{h}} \backslash B_{s_{h}}} f_{h}\left(D^{k} z_{h}\right)
$$

on the other hand we have

$$
\begin{aligned}
& \int_{B_{r_{h} \backslash B_{s_{h}}}} f_{h}\left(D^{k} z_{h}\right) \leq \int_{B_{r_{h}} \backslash B_{s_{h}}}\left|D^{k} z_{h}\right|^{2}+\lambda_{h}^{q-2}\left|D^{k} z_{h}\right|^{q} \\
& \leq c m^{-2 \rho}\left[f_{B_{r} \backslash B_{s}}\left(1+\sum_{l=0}^{k}\left|D^{l} v_{h}\right|^{2}\right)+\lambda_{h}^{\frac{q-2}{q} p} f_{B_{r} \backslash B_{s}}\left(1+\sum_{l=0}^{k}\left|D^{l} v_{h}\right|^{p}\right)\right]^{\frac{q}{2}}
\end{aligned}
$$

and then arguing as we did in Step 3 to bound $J_{h, 1}$ we get

$$
\underset{h}{\limsup } \int_{B_{r_{h}} \backslash B_{s_{h}}} f_{h}\left(D^{k} z_{h}\right) \leq C m^{-2 \rho}
$$

hence, letting $h \rightarrow \infty$ we get

$$
\liminf _{h} R_{h, 1} \geq-C m^{-2 \rho}
$$

We obtain that

$$
\begin{aligned}
R_{h, 2} & =\int_{B_{r_{h}} \backslash B_{s_{h}}} f_{h}\left(D^{k} z_{h}\right)-f_{h}\left(D^{k} \psi_{h}^{\epsilon}+D^{k} v_{r, s}^{\epsilon}\right) \\
& \geq-c \int_{B_{r_{h}} \backslash B_{s_{h}}}\left|D^{k} \psi_{h}^{\epsilon}+D^{k} v_{r, s}^{\epsilon}\right|^{2}+\lambda_{h}^{q-2}\left|D^{k} \psi_{h}^{\epsilon}+D^{k} v_{r, s}^{\epsilon}\right|^{q} \\
& \geq-c \int_{B_{r_{h}} \backslash B_{s_{h}}}\left|D^{k} z_{h}\right|^{2}+\lambda_{h}^{q-2}\left|D^{k} z_{h}\right|^{q}+\left|D^{k} v_{r, s}^{\epsilon}\right|^{2}+\lambda_{h}^{q-2}\left|D^{k} v_{r, s}^{\epsilon}\right|^{q} \\
& -c \int_{B_{r_{h}} \backslash B_{s_{h}}}\left(\sum_{l=0}^{k-1} \frac{m^{2(k-l)}}{(r-s)^{2(k-l)}} \mid D^{l}\left(z_{h}-\left.v_{r, s}^{\epsilon}\right|^{2}+\lambda_{h}^{q-2} \sum_{l=0}^{k-1} \frac{m^{q(k-l)}}{(r-s)^{q(k-l)}}\left|D^{l}\left(z_{h}-v_{r, s}^{\epsilon}\right)\right|^{q}\right)\right. \\
& =-S_{h, 1}-S_{h, 2}
\end{aligned}
$$

where we used the bound $r_{h}-s_{h} \geq \frac{r-s}{3 m}$. Denoting by $P_{l}$ the polynomial of degree $k-1$ such that

$$
\int_{B_{1}}\left(D^{l}\left(P_{l}-z_{h}\right)\right)=0
$$


for $l<k$, and setting

$$
p^{*}= \begin{cases}\frac{n p}{n-l p} & \text { if } p<\frac{n}{l} \\ r>p & \text { if } p \geq \frac{n}{l}\end{cases}
$$

since $q<p^{*}$, we get by $(23)$, for every $l=0, \ldots, k-1$

$$
\begin{aligned}
\int_{B_{1}} \lambda_{h}^{q-2}\left|D^{l} z_{h}\right|^{q} & \leq c \lambda_{h}^{q-2}\left\{\int_{B_{1}}\left|D^{l}\left(z_{h}-P_{l}\right)\right|^{q}+\left|D^{l}\left(P_{l}\right)\right|^{q}\right\} \\
& \leq c \lambda_{h}^{q-2}\left\{\left(\int_{B_{1}}\left|D^{l}\left(z_{h}-P_{l}\right)\right|^{p^{*}}\right)^{\frac{q}{p^{*}}}+\left(\int_{B_{1}}\left|D^{l}\left(P_{l}\right)\right|^{p^{*}}\right)^{\frac{q}{p^{*}}}\right\} \\
& \leq c \lambda_{h}^{q-2}\left(\int_{B_{1}}\left|D^{k} z_{h}\right|^{p}\right)^{\frac{q}{p}} \\
& \leq c \lambda_{h}^{\frac{2(q-p)}{p}}\left(\lambda_{h}^{p-2} \int_{B_{1}}\left|D^{k} z_{h}\right|^{p}\right)^{\frac{q}{p}} .
\end{aligned}
$$

Therefore, using (24), we obtain

$$
\limsup _{h \rightarrow \infty} S_{h, 2} \leq c \sum_{l=0}^{k-1} \frac{m^{2(k-l)}}{(r-s)^{2(k-l)}} \int_{B_{\frac{1}{2}}}\left|D^{l}\left(v_{r, s}-v_{r, s}^{\epsilon}\right)\right|^{2}
$$

To bound $S_{h, 1}$, observe that for every $h$

$$
\begin{gathered}
\int_{B_{r_{h}} \backslash B_{s_{h}}}\left|D^{k} v_{r, s}^{\epsilon}\right|^{2} \leq c \int_{B_{r} \backslash B_{s}}\left|D^{k} v_{r, s}\right|^{2}+c \int_{B_{\frac{1}{2}}}\left|D^{k} v_{r, s}-D^{k} v_{r, s}^{\epsilon}\right|^{2} \\
\quad \leq \liminf _{j} c \int_{B_{r} \backslash B_{s}}\left|D^{k} z_{j}\right|^{2}+c \int_{B_{\frac{1}{2}}}\left|D^{k} v_{r, s}-D^{k} v_{r, s}^{\epsilon}\right|^{2} \\
=c \liminf _{j} \int_{\left(B_{r} \backslash B_{s}\right) \backslash\left(B_{r_{j}} \backslash B_{s_{j}}\right)}\left|D^{k} v_{j}\right|^{2} \\
+c \limsup _{j} \int_{B_{r_{j}} \backslash B_{s_{j}}}\left|D^{k} z_{j}\right|^{2}+c \int_{B_{\frac{1}{2}}}\left|D^{k} v_{r, s}-D^{k} v_{r, s}^{\epsilon}\right|^{2} .
\end{gathered}
$$

We control the second integral as usual using Lemma 2.4, while the first is less or equal than $c \mu\left(B_{r} \backslash B_{s}\right)$.

Moreover we can estimate

$$
\int_{B_{r_{h}} \backslash B_{s_{h}}}\left|D^{k} z_{h}\right|^{2}+\lambda_{h}^{q-2}\left|D^{k} z_{h}\right|^{q}
$$

as we did in Step 3 to bound $J_{h, 1}$. Hence

$$
\begin{gathered}
\liminf _{h} R_{h, 2} \geq-c m^{-2 \rho}-c \mu\left(B_{r} \backslash B_{s}\right) \\
-c \int_{B_{\frac{1}{2}}}\left|D^{k} v_{r, s}-D^{k} v_{r, s}^{\epsilon}\right|^{2}-\sum_{l=0}^{k-1} \frac{m^{2(k-l)}}{(r-s)^{2(k-l)}} \int_{B_{\frac{1}{2}}}\left|D^{l}\left(v_{r, s}-v_{r, s}^{\epsilon}\right)\right|^{2} .
\end{gathered}
$$


To bound $R_{h, 3}$ we observe that

$$
f_{h}(A+B)-f_{h}(A)-f_{h}(B)=\int_{0}^{1} \int_{0}^{1} D^{2} f_{h}(s A+t B) A B \mathrm{~d} s \mathrm{~d} t
$$

and, by the definition of $f_{h}$,

$$
D^{2} f_{h}\left(s D^{k} v_{r, s}^{\epsilon}+t D^{k} \psi_{h}^{\epsilon}\right)=D^{2} f\left(A_{h}+s \lambda_{h} D^{k} v_{r, s}^{\epsilon}+t \lambda_{h} D^{k} \psi_{h}^{\epsilon}\right)
$$

is bounded and converges to $D^{2} f(A)$ a.e. Since

$$
R_{h, 3}=\int_{B_{r_{h}}} \mathrm{~d} x \int_{[0,1] \times[0,1]} D^{2} f\left(A_{h}+s \lambda_{h} D^{k} v_{r, s}^{\epsilon}+t \lambda_{h} D^{k} \psi_{h}^{\epsilon}\right) D^{k} v_{r, s}^{\epsilon} D^{k} \psi_{h}^{\epsilon} \mathrm{d} s \mathrm{~d} t
$$

and we may suppose that $\psi_{h}^{\epsilon} \rightarrow \psi^{\epsilon}$ weakly in $W^{k, 2}\left(B_{1}\right)$, and arguing as in the proof of (27), we have

$$
\int_{B_{1}}\left|D^{k} \psi^{\epsilon}\right|^{2} \leq \sum_{l=0}^{k-1} \frac{m^{2(k-l)}}{(r-s)^{2(k-l)}} \int_{B_{\frac{1}{2}}}\left|D^{l}\left(v_{r, s}-v_{r, s}^{\epsilon}\right)\right|^{2}+c \int_{B_{\frac{1}{2}}}\left|D^{k} v_{r, s}-D^{k} v_{r, s}^{\epsilon}\right|^{2} .
$$

Then we get easily

$$
\underset{h}{\limsup }\left|R_{h, 3}\right| \leq c(M)\left\|D^{k} v_{r, s}^{\epsilon}\right\|_{L^{2}\left(B_{\frac{1}{2}}\right)}|| D^{k} \psi^{\epsilon} \|_{L^{2}\left(B_{\frac{1}{2}}\right)} .
$$

To bound $R_{h, 4}$ we observe that

$$
R_{h, 4}=\int_{B_{r_{h}} \backslash B_{s}}\left[f_{h}\left(D^{k} v_{r, s}^{\epsilon}\right)-f_{h}\left(D^{k} v^{\epsilon}\right)\right] \geq-\int_{B_{r_{h}} \backslash B_{s-\epsilon}} f_{h}\left(D^{k} v^{\epsilon}\right) \geq-c\left|B_{r} \backslash B_{s-\epsilon}\right| .
$$

Then

$$
\liminf _{h} R_{h, 4} \geq-c\left|B_{r} \backslash B_{s-\epsilon}\right|
$$

Moreover (H3) implies

$$
\left|R_{h, 5}\right|=I_{h, r_{h}}\left(\psi_{h}^{\epsilon}\right)=\int_{B_{r_{h}}} f_{h}\left(D^{k} \psi_{h}^{\epsilon}\right) \geq \gamma \int_{B_{t}}\left(1+\lambda_{h}^{p-2}\left|D^{k} v^{\epsilon}-D^{k} v_{h}\right|^{p-2}\right)\left|D^{k} v^{\epsilon}-D^{k} v_{h}\right|^{2}
$$

for $\epsilon$ small enough.

Passing to a subsequence we may suppose that

$$
\underset{h}{\limsup } R_{h, 5}=\lim _{h} R_{h, 5} .
$$

Therefore returning to (25), from (26), (27), (28), (29) and (30) we get

$$
\begin{aligned}
& \liminf _{h}\left[I_{h, r}\left(v_{h}\right)-I_{h, r}\left(v^{\epsilon}\right)\right] \\
& \geq \gamma \limsup _{h} \int_{B_{s}}\left(1+\lambda_{h}^{p-2}\left|D^{k} v^{\epsilon}-D^{k} v_{h}\right|^{p-2}\right)\left|D^{k} v^{\epsilon}-D^{k} v_{h}\right|^{2}-c\left|B_{r} \backslash B_{s-\epsilon}\right|-c \mu\left(B_{r} \backslash B_{s}\right) \\
& \left.\quad-c|| D^{k} v_{r, s}^{\epsilon}||_{L^{2}\left(B_{\frac{1}{2}}\right.}\right)\left|D^{k} \psi^{\epsilon} \|_{L^{2}\left(B_{\frac{1}{2}}\right)}-c m^{-2 \rho}-\int_{B_{\frac{1}{2}}}\right| D v_{r, s}-\left.D v_{r, s}^{\epsilon}\right|^{2} \\
& \quad-c \sum_{l=0}^{k-1} \frac{m^{2(k-l)}}{(r-s)^{2(k-l)}} \int_{B_{\frac{1}{2}}} \mid D^{l}\left(v_{r, s}-\left.v_{r, s}^{\epsilon}\right|^{2} .\right.
\end{aligned}
$$


Passing to the limit as $\epsilon \rightarrow 0^{+}$we get easily

$$
\begin{aligned}
\liminf _{h}\left[I_{h, r}\left(v_{h}\right)\right. & \left.-I_{h, r}(v)\right] \\
& \geq \gamma \underset{h}{\limsup } \int_{B_{s}}\left(1+\lambda_{h}^{p-2}\left|D^{k} v-D^{k} v_{h}\right|^{p-2}\right)\left|D^{k} v-D^{k} v_{h}\right|^{2}-c\left|B_{r} \backslash B_{s}\right|-c \mu\left(B_{r} \backslash B_{s}\right)-c m^{-2 \rho}
\end{aligned}
$$

then passing to the limit as $m \rightarrow \infty$ and $s \rightarrow r$ we get

$$
\underset{h}{\limsup } \int_{B_{r}}\left|D^{k} v-D^{k} v_{h}\right|^{2}\left(1+\lambda_{h}^{p-2}\left|D^{k} v-D^{k} v_{h}\right|^{p-2}\right) \leq \lim _{h}\left[I_{h, r}\left(v_{h}\right)-I_{h, r}(v)\right] .
$$

Step 5. Conclusion. From the two previous steps we conclude that, for any $B_{\tau}$, with $0<\tau<\frac{1}{4}$

$$
\lim _{h} \int_{B_{\tau}}\left|D^{k} v-D^{k} v_{h}\right|^{2}\left(1+\lambda_{h}^{p-2}\left|D^{k} v-D^{k} v_{h}\right|^{p}\right)=0
$$

Now, from this equality and by (18) we get

$$
\begin{aligned}
\lim _{h} \frac{U\left(x_{h}, \tau r_{h}\right)}{\lambda_{h}^{2}} & =\lim _{h} \frac{1}{\lambda_{h}^{2}} f_{B_{\tau r_{h}}\left(x_{h}\right)}\left(\left|D^{k} u-\left(D^{k} u\right)_{\tau r_{h}}\right|^{2}+\left|D^{k} u-\left(D^{k} u\right)_{\tau r_{h}}\right|^{p}\right) \mathrm{d} x \\
& =\lim _{h} f_{B_{\tau}}\left(\left|D^{k} u-\left(D^{k} u\right)_{\tau}\right|^{2}+\lambda_{h}^{p-2}\left|D^{k} u-\left(D^{k} u\right)_{\tau}\right|^{p}\right) \mathrm{d} y \\
& =f_{B_{\tau}}\left(\left|D^{k} v-\left(D^{k} v\right)_{\tau}\right|^{2}\right) \mathrm{d} y \\
& \leq C_{M}^{*} \tau^{2}
\end{aligned}
$$

which contradicts (10) if we choose $C_{M}=2 C_{M}^{*}$.

The proof of Theorem 2.1 follows by Proposition 3.1 by a standard iteration argument, see [12].

\section{REFERENCES}

[1] E. Acerbi and N. Fusco, Partial regularity under anisotropic $(p, q)$ growth conditions. J. Diff. Eq. 107 (1994) $46-67$.

[2] M. Bildhauer, Convex variational problems. Linear, nearly linear and anisotropic growth conditions. Lect. Notes Math. 1818, Springer-Verlag, Berlin (2003).

[3] M. Bildhauer and M. Fuchs, Higher order variational problems with non-standard growth condition in dimension two: plates with obstacles. Ann. Acad. Sci. Fennicae Math. 26 (2001) 509-518.

[4] M. Carriero, A. Leaci and F. Tomarelli, Strong minimizers of Blake \& Zisserman functional. Ann. Scuola Norm. Sup. Pisa Cl. Sci. 15 (1997) 257-285.

[5] R. Choksi, R.V. Kohn and F. Otto, Domain branching in uniaxial ferromagnets: a scaling law for the minimum energy. Comm. Math. Phys. 201 (1999) 61-79.

[6] B. Dacorogna, Direct methods in the calculus of variations. Appl. Math. Sci. 78, Springer Verlag (1989).

[7] G. Dal Maso, I. Fonseca, G. Leoni and M. Morini, Higher order quasiconvexity reduces to quasiconvexity Arch. Rational Mech. Anal. 171 (2004) 55-81.

[8] L. Esposito, F. Leonetti and G. Mingione, Regularity results for minimizers of irregular integrals with $(p, q)$ growth. Forum Math. 14 (2002) 245-272.

[9] L. Esposito, F. Leonetti and G. Mingione, Sharp regularity for functionals with $(p, q)$ growth. J. Diff. Eq. 204 (2004) 5-55.

[10] I. Fonseca and J. Malý, Relaxation of multiple integrals in Sobolev spaces below the growth exponent for the energy density. Ann. Inst. H. Poincaré - Anal. Non Linéaire 14 (1997) 309-338. 
[11] I. Fonseca and J. Malý, From Jacobian to Hessian: distributional form and relaxation. Riv. Mat. Univ. Parma (7) (2005), Proc. Conf. "Trends in the Calculus of Variations", E. Acerbi and G. Mingione Eds., 45-74.

[12] M. Giaquinta, Multiple integrals in the calculus of variations and nonlinear elliptic systems. Ann. Math. Stud. 105 (1983), Princeton Univ. Press.

[13] M.Giaquinta, Growth conditions and regularity, a counterexample. Manu. Math. 59 (1987) 245-248.

[14] E. Giusti, Metodi diretti in calcolo delle variazioni. U.M.I. (1994).

[15] M. Guidorzi, A remark on partial regularity of minimizers of quasiconvex integrals of higher order. Rend. Ist. Mat di Trieste XXXII (2000) 1-24.

[16] M. Kronz, Partial regularity results for minimizers of quasiconvex functionals of higher order. Ann. Inst. H. Poincaré - Anal. Non Linéaire 19 (2002) 81-112.

[17] P. Marcellini, Un example de solution discontinue d'un probéme variationel dans le cas scalaire. Preprint Ist. U. Dini, Firenze (1987-1988).

[18] P. Marcellini, Regularity of minimizers of integrals of the calculus of Variations with non-standard growth conditions. Arch. Rat. Mech. Anal. 105 (1989) 267-284.

[19] P. Marcellini, Regularity and existence of solutions of elliptic equations with $(p, q)$ growth conditions. J. Diff. Eq. 90 (1991) $1-30$.

[20] P. Marcellini, Everywhere regularity for a class of elliptic systems without growth conditions. Ann. Scuola Normale Sup. Pisa, Cl. Sci. 23 (1996) 1-25.

[21] S. Müller and V. Šverák, Convex integration for Lipschitz mappings and counterexamples to regularity. Ann. of Math. 157 (2003) 715-742.

[22] A. Passarelli di Napoli and F. Siepe, A regularity result for a class of anisotropic systems. Rend. Ist. Mat di Trieste (1997) 13-31. 